\title{
Nonadiabatic Channels in the Superconducting Pairing of Fullerides
}

\author{
E. Cappelluti ${ }^{1}$, C. Grimaldi ${ }^{2}$, L. Pietronero ${ }^{1}$, and S. Strässler ${ }^{2}$ \\ 1 Dipartimento di Fisica, Universitá di Roma "La Sapienza", Piazzale A. Moro, 2, 00185 Roma, Italy \\ and Istituto Nazionale Fisica della Materia, Unitá di Roma 1, Italy \\ ${ }^{2}$ École Polytechnique Fédérale de Lausanne, Département de Microtechnique IPM, CH-1015 Lausanne, Switzerland
}

(October 23, 2018)

\begin{abstract}
We show the intrinsic inconsistency of the conventional phonon mediated theory of superconductivity in relation to the observed properties of $\mathrm{Rb}_{3} \mathrm{C}_{60}$. The recent, highly accurate measurement of the carbon isotope coefficient $\alpha_{\mathrm{C}}=0.21$, together with the high value of $T_{c}(30 \mathrm{~K})$ and the very small Fermi energy $E_{\mathrm{F}}(0.25 \mathrm{eV})$, unavoidably implies the opening of nonadiabatic channels in the superconducting pairing. We estimate these effects and show that they are actually the key elements for the high value of $T_{c}$ in these materials compared to the very low values of graphite intercalation compounds.

PACS number(s): 74.20.-z, 74.70.Wz, 71.38.+i
\end{abstract}

One of the most striking evidences of the phonon role in high-temperature superconductivity of alkali-doped $\mathrm{C}_{60}$ compounds is the observation of nonzero isotope effect on the value of the critical temperature $T_{c}$ [1]. However, the large spread of the reported values of the carbon isotope coefficient $\alpha_{\mathrm{C}}=-d \ln T_{c} / d \ln M$ [2], where $M$ is the isotopic mass, has prevented to settle a definitive and self-consistent picture. This long standing uncertainty has been solved only recently for the compound $\mathrm{Rb}_{3} \mathrm{C}_{60}$ $\left(T_{c} \simeq 30 \mathrm{~K}\right)$. Resistive measurements on $99 \%$ enriched ${ }^{13} \mathrm{C}$ single-crystals have permitted in fact to establish $\alpha_{\mathrm{C}}=0.21$ with high precision [3].

The knowledge of the accurate value of $\alpha_{\mathrm{C}}$ is an important element to establish not only the important role of phonons, but also to test the self-consistency of the Migdal-Eliashberg (ME) theory of superconductivity [4, 5] in alkali-doped fullerenes. The measured values $\alpha_{\mathrm{C}}=0.21$ and $T_{c}=30 \mathrm{~K}$ can be used to extract the microscopic quantities involved in the superconducting pairing. For example, in Ref. [3], $T_{c}=30 \mathrm{~K}$ and $\alpha_{\mathrm{C}}=0.21$ are interpreted within the conventional ME theory by $\lambda=0.9, \omega_{\mathrm{ln}}=1360 \mathrm{~K}$ and $\mu^{*}=0.22$, where $\lambda$ is the electron-phonon coupling constant, $\omega_{\ln }$ is the logarithmic phonon frequency and $\mu^{*}$ is the Coulomb pseudopotential [6]. According to this standard analysis, the high value of $T_{c}$ in alkali-doped $\mathrm{C}_{60}$ compounds is merely due to a strong electron-phonon coupling to the highest intramolecular phonon modes. These results should be compared with the graphite intercalation compounds (GIC) where $T_{c} \simeq 0.2 \mathrm{~K} 7$ is explained by a moderate coupling $(\lambda \simeq 0.3)$ to similar high energy phonon modes. Current theories claim that the big difference between the electron-phonon coupling in fullerides compared with graphite intercalation compounds arises from the finite curvature of the $\mathrm{C}_{60}$ molecule [8]. In this perspective, therefore, $\mathrm{Rb}_{3} \mathrm{C}_{60}$ is just an ordinary strongcoupling superconductor described by the conventional adiabatic ME framework.

In this Letter instead we demonstrate the intrinsic in- consistency of the standard ME theory in $\mathrm{Rb}_{3} \mathrm{C}_{60}$. This conventional description is in fact invalidated by the extremely low value of the Fermi energy $E_{\mathrm{F}} \simeq 0.25 \mathrm{eV}$ $\simeq 2900 \mathrm{~K}$ characteristic of the fullerene compounds [1]. We show that the whole range of $\lambda-\omega_{\mathrm{ph}}$ values which fit $T_{c}=30 \mathrm{~K}$ and $\alpha_{\mathrm{C}}=0.21$ through the solution of the standard $\mathrm{ME}$ equations implies a Migdal parameter $\lambda \omega_{\mathrm{ph}} / E_{\mathrm{F}}$ larger than 0.4 , instead of being zero as assumed by the ME theory [4.5]. This situation inevitably leads to the breakdown of Migdal's theorem and to the opening of nonadiabatic channels in the superconducting pairing [9]. By solving the nonadiabatic equations [10, 11, we estimate these effects and show that they are actually the key elements for the high values of $T_{c}$ in the fullerene compounds.

We now discuss why the experimental data $T_{c}=30 \mathrm{~K}$ and $\alpha_{\mathrm{C}}=0.21$ [3] are inconsistent with respect to the ME theory. To simulate the coupling of the electrons to the different intramolecular phonon modes, we consider an electron-phonon spectral function $\alpha^{2} F(\omega)$ modeled as a rectangle of width $\Delta \omega_{0}$ and centered at $\omega_{0}$. For $\Delta \omega_{0}=0$, $\alpha^{2} F(\omega)$ reduces to a single Einstein $\delta$-peak while for $\Delta \omega_{0}>0$ it becomes broadened. The electron-phonon coupling constant is determined by the usual relation $\lambda=$ $2 \int d \omega \alpha^{2} F(\omega) / \omega$. The Coulomb pseudopotential is taken to have the standard form $\mu^{*}=\mu /\left[1+\mu \ln \left(\omega_{c} / \omega_{\max }\right)\right]$, where $\omega_{\max }=\omega_{0}+\Delta \omega_{0} / 2$ is the maximum phonon frequency, $\mu$ is the screened Coulomb parameter and $\omega_{c}$ is the high-frequency cut-off. According to whether it is the entire set of $\pi$-bands [12] or rather only the narrow conducting $t_{1 \mathrm{u}}$ band 13 which contributes to the dynamical screening, we shall consider $\omega_{c}=5 \omega_{\max }$ or $\omega_{c}=E_{\mathrm{F}}=2900 \mathrm{~K}$, respectively. For different values of $\Delta \omega_{0} / \omega_{0}$ we then solve numerically the ME equations to find the values of $\lambda, \omega_{0}$, and $\mu$ (or $\mu^{*}$ ) which reproduce the experimental data $T_{c}=30 \mathrm{~K}$ and $\alpha_{\mathrm{C}}=0.21$.

In Fig. 1 1 we show the calculated $\mu$ and $\mu^{*}$ (top panel) and $\omega_{0}$ (lower panel) as function of $\lambda$ for $\Delta \omega_{0} / \omega_{0}=0$ (solid lines) and $\Delta \omega_{0} / \omega_{0}=1$ (dashed lines) with $\omega_{c}=$ 


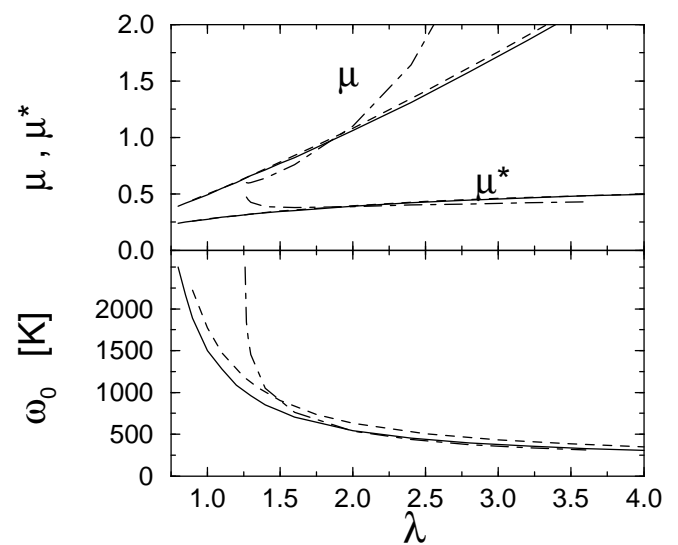

FIG. 1. Plot of the Coulomb parameters $\mu$ and $\mu^{*}$ (upper panel) and of the central phonon frequency $\omega_{0}$ (lower panel) obtained by the standard $\mathrm{ME}$ equations constrained to have $T_{c}=30 \mathrm{~K}$ and $\alpha_{\mathrm{C}}=0.21$. Solid lines: $\Delta \omega_{0} / \omega_{0}=0$, $\omega_{c}=5 \omega_{\max } ;$ dashed lines: $\Delta \omega_{0} / \omega_{0}=1, \omega_{c}=5 \omega_{\max }$; dot-dashed lines: $\Delta \omega_{0} / \omega_{0}=0, \omega_{c}=E_{\mathrm{F}}$.

$5 \omega_{\max }$. The main point of Fig. 1 is that the calculated $\omega_{0}$ depends strongly on the electron-phonon constant $\lambda$. For large values of $\lambda, T_{c}=30 \mathrm{~K}$ and $\alpha_{\mathrm{C}}=0.21$ are reproduced only for quite small phonon frequencies while decreasing $\lambda$ quickly enhances $\omega_{0}$. The $\mathrm{C}_{60}$ phonon spectrum however is limited by a maximum frequency of $\sim 2300 \mathrm{~K}$ [1,14] and this settles a lower limit for the allowed values of $\lambda$. In fact, from the lower panel of Fig. 1, it is clear that for $\lambda$ smaller than 1 the corresponding $\omega_{\max }=\omega_{0}+\Delta \omega_{0} / 2$ rapidly exceeds $2300 \mathrm{~K}$ signalling that the solution of the ME equations falls well outside the range of applicability for the fullerene compounds.

A further interesting point is that the effect of the spectral broadening $\left(\Delta \omega_{0}>0\right)$ is of secondary importance to the overall behavior, suggesting that the results are only weakly affected by the detailed structure of $\alpha^{2} F(\omega)$. In fact, for $\lambda=0.9$ we find $\mu^{*} \simeq 0.25, \omega_{\ln } \simeq 1873$ $\mathrm{K}\left(\Delta \omega_{0}=0\right)$ and $\omega_{\ln } \simeq 1555 \mathrm{~K}\left(\Delta \omega_{0}=\omega_{0}\right)$, where $\omega_{\ln }=\exp \left[(2 / \lambda) \int d \omega \ln \omega \alpha^{2} F(\omega) / \omega\right]$ is the logarithmic phonon frequency which, for the rectangular model here considered, is given by $\omega_{\ln }=\left[\omega_{0}^{2}-\left(\Delta \omega_{0} / 2\right)^{2}\right]^{1 / 2}$. These values are consistent with those reported in Ref. [3] $\left(\lambda \simeq 0.9, \mu^{*} \simeq 0.22, \omega_{\ln } \simeq 1360 \mathrm{~K}\right)$ where a phonon spectrum obtained by $a b$ initio calculations has been used to fit $T_{c}=30 \mathrm{~K}$ and $\alpha_{\mathrm{C}}=0.21$.

In Fig. 11 we show also the results for $\Delta \omega_{0}=0$ and $\omega_{c}=$ $E_{\mathrm{F}}$ (dot-dashed lines). Due to the reduced dynamical screening of the Coulomb repulsion, the increase of $\omega_{0}$ is much steeper than the previous cases until at $\lambda \simeq 1.25$ we find $\omega_{0} \simeq E_{\mathrm{F}}$ and $\mu^{*} \simeq \mu$ so that it is no longer possible to have $\alpha_{\mathrm{C}}$ smaller than the BCS value 0.5.

We now address the consistency of the data of Fig. 1 with the standard ME theory 娌5. The assumption at the basis of the ME framework is Migdal's theorem which states that, as long as the phonons have a much

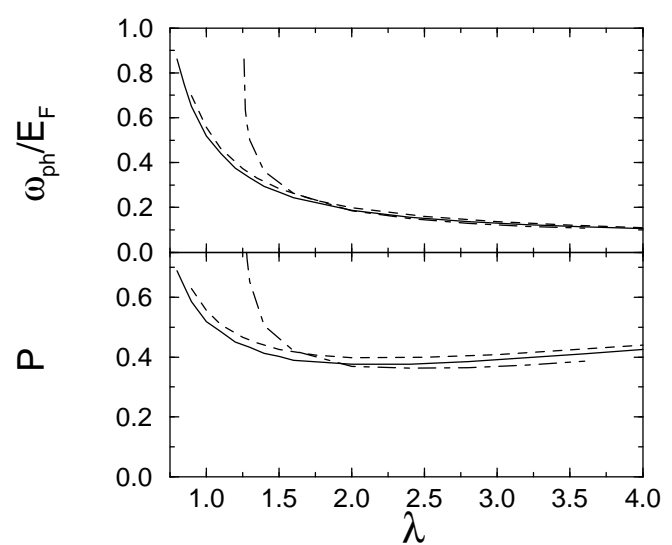

FIG. 2. Values of $\omega_{\mathrm{ph}} / E_{F}$ (upper panel) and of $P=\lambda \omega_{\mathrm{ph}} / E_{F}$ (lower panel) extracted from the data of Fig. 1 with $E_{F}=0.25 \mathrm{eV}=2900 \mathrm{~K}$. Solid lines: Einstein phonon spectrum; dashed lines: broad spectrum with $\Delta \omega_{0} / \omega_{0}=1$, dot-dashed lines: Einstein phonon spectrum $\Delta \omega_{0} / \omega_{0}=1$ with $\omega_{c}=E_{\mathrm{F}}$. The Migdal-Eliashberg theory holds true only when $\omega_{\mathrm{ph}} / E_{F} \ll 1$ and $P \ll 1$.

slower dynamics than that of the electrons, the nonadiabatic electron-phonon interference effects (vertex processes) can be neglected [- This condition is well satisfied in conventional superconductors since their Fermi energy is of order $E_{F} \sim 10 \mathrm{eV} \sim 10^{5} \mathrm{~K}$ while the highest phonon frequencies are usually less than $\sim 50 \mathrm{meV} \sim 60$ $\mathrm{K}$ [6]. However, the alkali-doped fullerene compounds are molecular solids characterized by very narrow conduction electron bands of width of only $W \simeq 0.5 \mathrm{eV}$ [1]. In $\mathrm{Rb}_{3} \mathrm{C}_{60}$ (as in the other $\mathrm{A}_{3} \mathrm{C}_{60}$ compounds) the conduction band is half-filled by electrons and the corresponding Fermi energy is $E_{F} \simeq 0.25 \mathrm{eV}=2900 \mathrm{~K}$, while the maximum phonon frequency is $\sim 2300 \mathrm{~K}$. In principle, therefore, there is no reason to expect Migdal's theorem to be applicable in fullerene compounds, unless the main interaction is with the lowest $\mathrm{C}_{60}$ phonon modes $(\sim 400 \mathrm{~K})$ via however a rather weak coupling.

We can test whether the data of Fig. 1 are consistent with Migdal's theorem by evaluating the order of magnitude $(P)$ of the first nonadiabatic electron-phonon vertex correction. By following the same reasonings of Migdal [4, 10], this is given by:

$$
P=2 \int_{0}^{+\infty} d \omega \frac{\alpha^{2} F(\omega)}{E_{F}} \equiv \lambda \frac{\omega_{\mathrm{ph}}}{E_{F}}
$$

where for the rectangular model we considered the average phonon frequency $\omega_{\mathrm{ph}}$ reduces to

$$
\omega_{\mathrm{ph}}=\frac{2}{\lambda} \int_{0}^{+\infty} d \omega \alpha^{2} F(\omega)=\frac{\Delta \omega_{0}}{\ln \left(\frac{\omega_{0}+\Delta \omega_{0} / 2}{\omega_{0}-\Delta \omega_{0} / 2}\right)} .
$$

When $P \rightarrow 0$ the nonadiabatic interferences are negligible and the ME theory holds true; on the contrary, sizeable values of $P$ signal the breakdown of the standard 
theory. In Fig. 2 we show the values of $\omega_{\mathrm{ph}} / E_{F}$ (upper panel) and of $P$ (lower panel) extracted from the $\omega_{0}$ values reported in Fig. 11 and by setting $E_{F}=2900 \mathrm{~K}$. As expected from the overall trend of $\omega_{0}$ vs $\lambda$ plotted in Fig. 1, the adiabatic ratio $\omega_{\mathrm{ph}} / E_{F}$ is large and close to unity for $\lambda<1$, while it rapidly decreases to $\omega_{\mathrm{ph}} / E_{F} \simeq 0.1$ for large values of $\lambda$. One could therefore argue that adiabaticity is guaranteed for very large electron-phonon couplings and that in this regime the ME framework is valid. This is however incorrect because according to (11) the vertex correction is proportional to $\lambda$ so that, as shown in the lower panel of Fig. $2, P$ is never negligible. Note that the claimed value $\lambda \simeq 0.9$ [3] corresponds to $\omega_{\mathrm{ph}} / E_{F}>0.7$ and a minimum $P>0.6$, i.e., the vertex correction is comparable to unity.

From Figs. 1 and 2 we conclude therefore that the conventional phonon-mediated superconductivity is not a complete and self-consistent picture of $\mathrm{Rb}_{3} \mathrm{C}_{60}$ since the values of $\lambda$ and $\omega_{0}$ needed to fit $T_{c}=30 \mathrm{~K}$ and $\alpha_{\mathrm{c}}=0.21$ strongly violate Migdal's theorem. This conclusion holds true even when electron-phonon spectra more structured than the rectangular one are used to fit the data of Ref. [3]. By adding a $\delta$-peak centered at $\omega=\omega_{1}$ to a rectangular spectrum of width covering the whole intramolecular modes, we have in fact simulated additional contributions from low-frequency $\left(\omega_{1} \simeq 50 \mathrm{~K}\right) \mathrm{C}_{60}-\mathrm{C}_{60}$ phonon modes [15], and from an enhanced coupling to soft intramolecular modes 16] $\left(\omega_{1} \simeq 400-600 \mathrm{~K}\right)$ possibly related to dynamical Jahn-Teller effects 17]. We find that $T_{c}=30$ $\mathrm{K}$ and $\alpha_{\mathrm{C}}=0.21$ imply $P>0.4$ when $\omega_{1}=50 \mathrm{~K}$ and $P>0.45$ when $\omega_{1}=400 \mathrm{~K}$ (further details will be presented elsewhere).

The above results point out that, if superconductivity in $\mathrm{Rb}_{3} \mathrm{C}_{60}$ is mediated by phonons, a consistent description of its superconducting properties should be sought beyond the ME theory. More precisely, the low value of $E_{F}$ indicates that the adiabatic hypothesis and Migdal's theorem should be abandoned from the start and that the theory should be formulated by allowing $\omega_{\mathrm{ph}} / E_{F}$ to have values sensibly larger than zero. This naturally leads to nonadiabatic interference effects in the electron-phonon scattering which can significantly modify both the normal and superconducting properties with respect to the ME phenomenology [9].

Indeed, characteristic effects of the nonadiabatic vertex corrections are predicted to be observable in several quantities, like $T_{c}$ and its the isotope coefficient [9, 10], the reduction rate of $T_{c}$ itself upon disorder 11], the effective electronic mass $m^{*}$ [18], the Pauli susceptibility [19. A peculiar feature of the nonadiabatic processes is to produce, under some conditions, constructive electronphonon interference in the particle-particle channel leading to an enhancement of $T_{c}$ [9, 10]. Hence $T_{c}=30 \mathrm{~K}$, for a given phonon spectrum, can be achieved by much smaller values of $\lambda$ than needed in conventional ME theory. Favourable conditions to this trend are expected in

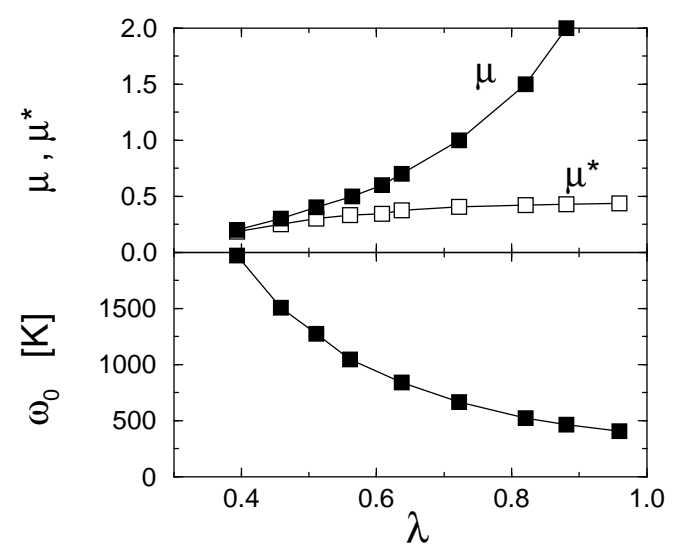

FIG. 3. Plot of $\mu, \mu^{*}$ and $\omega_{0}$ calculated by the nonadiabatic theory as solutions of $T_{c}=30 \mathrm{~K}$ and $\alpha_{\mathrm{C}}=0.21$. Note that the range of values for $\lambda$ is now much smaller and realistic with respect to those of Fig. 1.

materials with strong electronic correlation, as fullerenes: strong local repulsion suppresses short-range interactions (large q's in Fourier space) and favours forward small-q scattering 220.21] for which electron-phonon vertex processes become attractive.

Now we re-analyze the experimental constraints of $\mathrm{Rb}_{3} \mathrm{C}_{60}, T_{c}=30 \mathrm{~K}$ and $\alpha_{\mathrm{C}}=0.21$, in the context of the nonadiabatic theory of superconductivity. We show that the inconsistencies of the results derived by ME theory are naturally solved when the same experimental data are coherently analyzed in the nonadiabatic regime.

Explicit analytical and diagrammatic equations of the nonadiabatic theory of superconductivity have been outlined in previous works 911 and, for sake of shortness, they will be here omitted. A set of generalized Eliashberg equations in nonadiabatic regime is constructed by following a perturbative approach based on the Migdal parameter $P$. The consistency of such a perturbative scheme is discussed below. We simplify the phonon spectrum by assuming a dispersionless Einstein phonon with energy $\omega_{0}$. We note however that our results, as also shown in Figs. 1 and 2, are only weakly affected by the specific shape of $\alpha^{2} F(\omega)$. Electronic correlation is taken into account by a cut-off $q_{c}$ on the electron-phonon exchanged momenta, which selects forward scattering, where stronger the correlation smaller $q_{c}$.

In Fig. 3 we show the phonon frequency $\omega_{0}$, the statically and dinamically screened Coulomb repulsion, respectively $\mu$ and $\mu^{*}$, vs. $\lambda$ obtained in the nonadiabatic theory to reproduce $T_{c}=30 \mathrm{~K}$ and $\alpha_{\mathrm{C}}=0.21$. The parameter $q_{c}$ has been chosen $q_{c}=0.2 k_{\mathrm{F}}\left(k_{\mathrm{F}}\right.$ the Fermi vector), an appropriate value for a strongly correlated system, and the dinamical screening of $\mu$ has been considered to be provided by the $t_{1 \mathrm{u}}$ electrons [13].

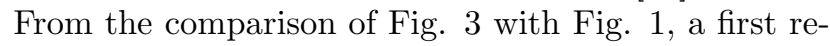
markable difference lies in the distinct ranges of electronphonon couplings needed to reproduce the $\mathrm{Rb}_{3} \mathrm{C}_{60}$ data. 


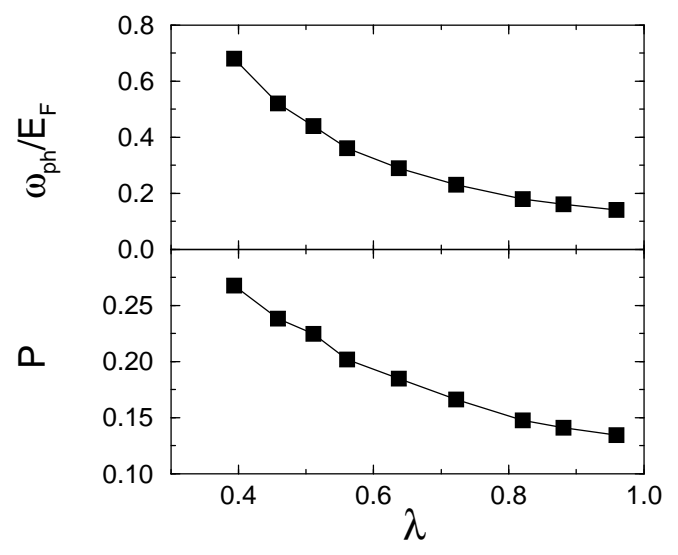

FIG. 4. Adiabatic ratio $\omega_{\mathrm{ph}} / E_{F}$ and vertex correction magnitude $P$ obtained by the nonadiabatic solutions of Fig. 3

In fact, the conventional ME theory predicts $\lambda \gtrsim 1$ (Fig. 11), while the nonadiabatic analysis yields $\lambda \lesssim 1$ (Fig. 33). But the most striking difference is that, if we now take the parameters obtained by the generalized theory and use them in the standard ME theory, these would give a very low value of $T_{c}$, less than $1 \mathrm{~K}$ or even zero. This result is now perfectly compatible with the GIC superconductors, for which Migdal's theorem holds true, and it clarifies that the high $T_{c}$ values of the fullerides are essentially due to constructive nonadiabatic interference effects rather than to a very large value of $\lambda$. In our perspective, therefore, the origin of the enhancement from $T_{c} \simeq 0.2 \mathrm{~K}$ in GIC to $T_{c} \simeq 20-30 \mathrm{~K}$ in fullerene compounds stems mainly from the opening the nonadiabatic channels in the electron-phonon interaction, rather than from a $\sim 300 \%$ enhancement of $\lambda$.

We address now the consistency of the perturbative scheme with the nonadiabatic solutions for $\mathrm{Rb}_{3} \mathrm{C}_{60}$. In Fig. 14 we show the adiabatic ratio $\omega_{\mathrm{ph}} / E_{F}\left(\omega_{\mathrm{ph}}=\omega_{0}\right)$ and the Migdal's parameter $P$ extracted from the data reported in Fig. 3. The large value of $\omega_{\mathrm{ph}} / E_{F}$ shown in the upper panel points out again the breakdown of Migdal's theorem and consequently the need of the inclusion of the nonadiabatic vertex corrections. The magnitude of the vertex corrections $P \sim 0.2$, certainly not negligible, is however small enough to support a perturbative approach in $P$ [9.10]. Note moreover that, according to the comparison with exact results for the single-electron Holstein model [22], for weak couplings the system is away from polaron formation and that the perturbative scheme is well defined.

In conclusion, we have investigated the validity of Migdal-Eliashberg theory of superconductivity in $\mathrm{Rb}_{3} \mathrm{C}_{60}$ by analyzing the constraints imposed by recent experimental data, namely the critical temperature $T_{c}=30$ $\mathrm{K}$ and the isotope effect $\alpha_{\mathrm{C}}=0.21$. We have found that the values of $\lambda$ and $\omega_{\mathrm{ph}}$ needed to reproduce the experimental data, together with the very low value of the Fermi energy, strongly violate Migdal's theorem and are therefore inconsistent with the ME framework. This situation unavoidably leads to the opening of nonadiabatic channels in the electron-phonon pairing which we argue to play the primary role for the high values of $T_{c}$ in fullerene compounds. Finally, we stress the importance of peculiar nonadiabatic effects in both superconducting [11] and normal state properties [18 19 of fullerene compounds. Experiments in this direction are therefore of great interest.

[1] O. Gunnarsson, Rev. Mod. Phys. 69, 575 (1997)

[2] A. A. Zakhidov et al., Phys. Lett. a 164, 355 (1992); C. -C. Chen and C. M. Lieber, Science 259, 655 (1993); P. Auban-Senzier et al., Synth. Met. 55-57, 3027 (1993).

[3] M. S. Fuhrer, K. Cherrey, A. Zettl, M. L. Cohen, and V. H. Crespi, Phys. Rev. Lett. 83404 (1999).

[4] A. B. Migdal, Zh. Éksp. Teor. Fiz. 34, 1438 (1958) [Sov. Phys. JETP 7, 996 (1958)].

[5] G. M. Eliashberg, Zh. Éksp. Teor. Fiz. 38, 966 (1960) [Sov. Phys. JETP 11, 696 (1960)].

[6] J. P. Carbotte, Rev. Mod. Phys. 62, 1027 (1990).

[7] I. T. Belash, A. D. Bronnikov, O. V. Zharikov, and A. V. Palnichenko, Synth. Met. 36, 283 (1990).

[8] P. J. Benning, J. L. Martins, J. H. Weaver, L. P. F. Chibante, and R. E. Smalley, Science 252, 1417 (1991); M. Schluter, M. Lannoo, M. Needels, G. A. Baraff, and D. Tománek, Phys. Rev. Lett. 68, 526 (1992).

[9] C. Grimaldi, L. Pietronero, and S. Strässler, Phys. Rev. Lett. 75, 1158 (1995).

[10] L. Pietronero, S. Strässler, and C. Grimaldi, Phys. Rev. B 52, 10516 (1995); C. Grimaldi, L. Pietronero, and S. Strässler, Phys. Rev. B 52, 10530 (1995).

[11] M. Scattoni, C. Grimaldi, and L. Pietronero, Europhys. Lett. 47, 588 (1999).

[12] C. M. Varma, J. Zaanen, and K. Raghavachari, Science 25, 989 (1991).

[13] E. Koch, O. Gunnarsson, and R. M. Martin, Phys. Rev. Lett. 83, 620 (1999).

[14] A. F. Hebard, Phys. Today 45, No. 11, 26 (1992).

[15] I. I. Mazin, O. V. Dolgov, A. Golubov, and S. V. Shulga, Phys. Rev. B 47, 538 (1993).

[16] K. Prassides et al., Nature 354, 462 (1991).

[17] V. M. Loktev and É. A. Pashitskiǔ, Pis'ma Zh. Éksp. Teor. Fiz. 55, 465 (1992) [JETP Lett. 55, 478 (1992)]; ibid. Zh. Éksp. Teor. Fiz. 103, 594 (1993) [Sov. Phys. JETP 76, 297 (1993)].

[18] C. Grimaldi, E. Cappelluti, and L. Pietronero, Europhys. Lett. 42, 667 (1998).

[19] C. Grimaldi and L. Pietronero, Europhys. Lett. 47, 681 (1999).

[20] M. L. Kulić and R. Zeyher, Phys. Rev. B 49, 4395 (1994); R. Zeyher and M. L. Kulić, Phys. Rev. B 53, 2850 (1996).

[21] M. Grilli and C. Castellani, Phys. Rev. B 50, 16880 (1994). 
[22] M. Capone, S. Ciuchi, and C. Grimaldi, Europhys. Lett. 42, 523 (1998). 